\title{
A Versatile Method for Correlated Imaging and Stitching of the Outer Surface of Cylindrically-Shaped Samples
}

\author{
Xuejun Wang ${ }^{1}$ \\ 1. Research Analytical, Nalco Water, An Ecolab Company, Naperville, IL
}

It is challenging to stitch together the entire outer surface of cylindrically-shaped samples. Quite often, to do so requires an expensive rotating motor with specialized instrumentation. This paper presents a simple but versatile method to image and stitch whole outer surface of cylindrically-shaped samples. This method is based on transition to rotation conversion as shown in Figure 1. Briefly, an objective is focused on the top of a cylindrically-shaped sample. The objective can move in the $\mathrm{Z}$ direction but not in the XY direction. A barrier off the stage is put at one side of the cylindrically-shaped sample, thus when the motorized stage is moved towards the barrier the cylindrically-shaped sample will rotate accordingly. In principle, any microscope with a motorized stage and stitching capabilities can utilize this method.

As an example, a corroded cylindrical sample was imaged and stitched using a Keyence digital microscope. The high quality color images reveal much higher sample detail than a regular digital camera. The same sample was cleaned and the images stitched with white light interferometry to provide 3D topographical information. The color image before cleaning and the 3D topographical image after cleaning were correlated using ImageJ/Fiji software. Figure 2 shows correlated images of a selected region. The color image before cleaning and topography image after cleaning are correlated by registering a few known landmarks followed by rotation, transition and scale adjustment. Based on the correlated image, the pit depth is proportional to the size of corrosion product before cleaning. In addition, there are two scratches on the cleaned sample. These scratches are not apparent on the corroded sample, suggesting that these scratches do not have enormous effect on corrosion. Overall color image can provide better contrast for tiny pits and is useful to get insight about corrosion mechanism; topography image after cleaning can provide more quantitative pit depth information. It is beneficial to compare them side by side for more complete understanding of the corrosion event. 


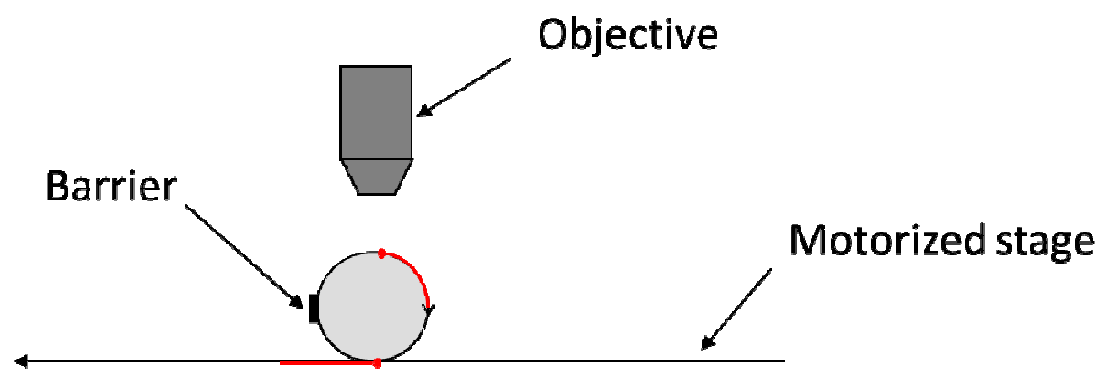

Figure 1. Schematic diagram of transition to rotation conversion
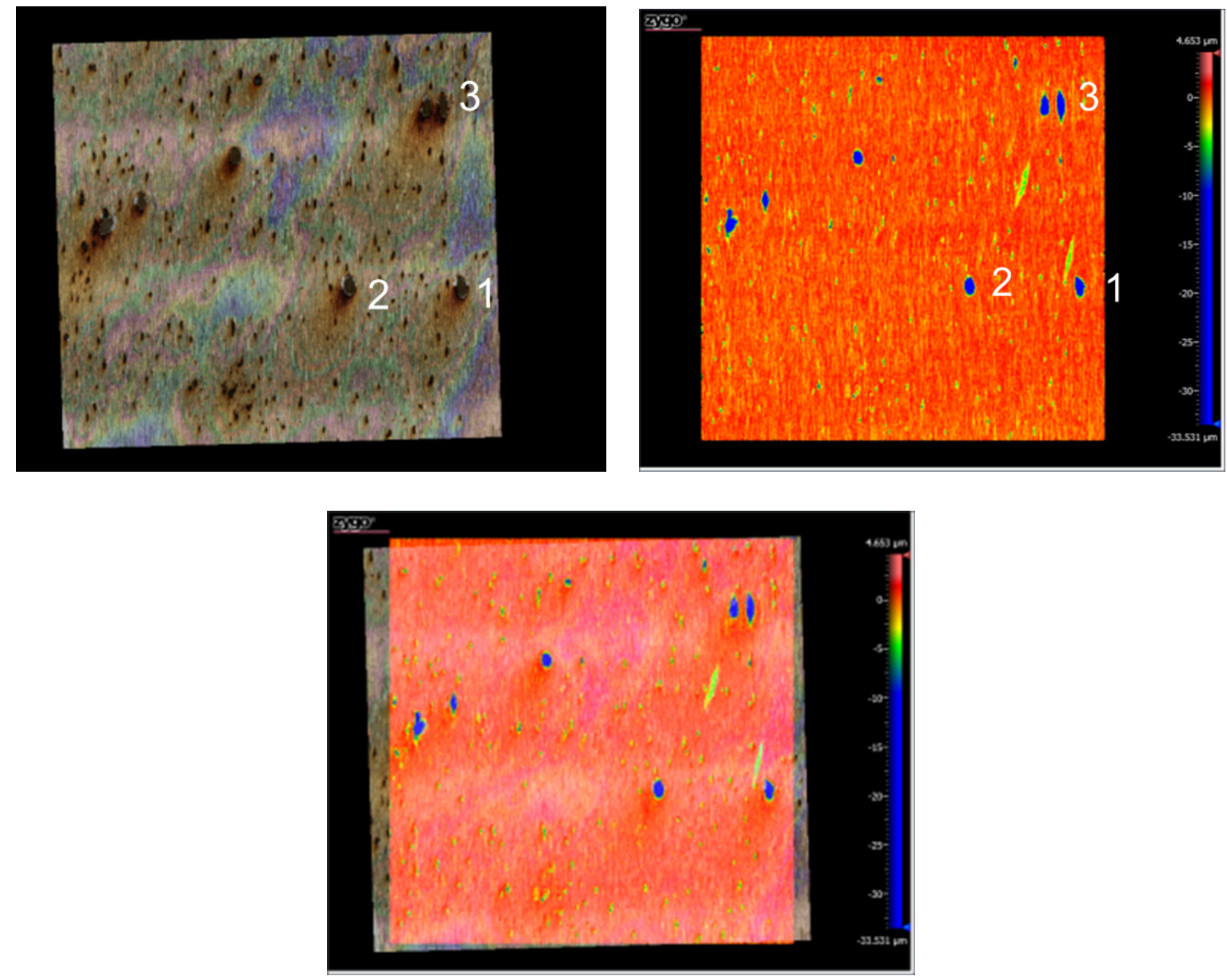

Figure 2. Correlated imaging: color image before cleaning (left), topography image after cleaning (right) and their overlaid image (bottom). 